\title{
A Communication-less Solution for Transient Frequency Drift Compensation on Weak Microgrids using a D-Statcom with an Energy Storage System
}

\author{
Ángel Navarro-Rodríguez, Pablo García, Ramy Georgious and Jorge García \\ Dept.of Elec., Computer \& System Engineering \\ University of Oviedo \\ Gijón, 33204, Spain \\ Email: navarroangel@uniovi.es, garciafpablo@uniovi.es, georgiousramy@uniovi.es, garciajorge@uniovi.es
}

\begin{abstract}
This paper proposes a solution for the transient grid frequency drift that occurs when active power transients take place in weak grids. Microgrids (MGs) are by definition weak grids, exhibiting a low inertia and high impedance which make them prone to contingencies that compromise the grid quality and stability. Particularly, the low inertia of the generators coupled to a MG could make the rotor speed to be affected by load changes, thus affecting the grid frequency. When compared with previous works, this research proposes a method for the estimation of the steady state reference frequency and the use of a Luenberger type observer for the estimation of the load current, leading to an improvement in the dynamics and the mitigation of the compensator phase lag due to the grid frequency estimator. Different control strategies are compared during the analytical discussion and validated through experimental results.
\end{abstract}

\section{INTRODUCTION}

The weakness and stability problems associated to a $\mathrm{MG}$ have been considered since the apparition of this grid concept, demanding significant research interests. Studies for different types of contingencies have been carried out with the aim of mitigation of unbalances, harmonics, frequency variations, and power quality improvement [1]-[3]. The most extended solution for power quality enhancement involving active power exchange in DG's and MGs, is referred in the literature as Distribution Static Synchronous Compensator (D-STATCOM) with ESS, or simply ESS [4], [5].

Several examples can be found about the use of ESS assisting power generation in DG's for frequency profile enhancement [6], [7]. Nevertheless, in these applications, the ESS works coordinated with a power plant using a communication link, providing access to the system parameters and variables. Often, the ESS participates both in the power share during steady state and transients. In the specific application of dynamic compensation it is worth mentioning the study in

The present work has been partially supported by the predoctoral grants program Severo Ochoa for the formation in research and university teaching of Principado de Asturias PCTI-FICYT under the grant ID BP14-135. This work also was supported in part by the Research, Technological Development and Innovation Program Oriented to the Society Challenges of the Spanish Ministry of Economy and Competitiveness under grant ENE2013-44245-R and by the European Union through ERFD Structural Funds (FEDER).
[3], where an ESS system was proposed for frequency compensation limited to the operation during transients. However such a solution presented a very bounded conditions. It relays on the communication with the generation system and the applied droop control may lead to insufficient performance in other applications. Being a relatively novel concept, the communication-less dynamic frequency drift compensation on MG's has been already presented, being one of the most studied solution the Virtual Inertia (VI) concept [8].

The absence of communication leads to three main constraints : 1) The use of grid frequency feedback based control requires the estimation of the frequency. Methods as Phase Lock Loop (PLL) [9], [10], based on angle estimation, or more robust techniques as Frequency Lock Loop (FLL) [11], focused on frequency estimation, can be used. However, they are characterized by relatively slow dynamics which limits the compensator controller bandwidth. 2) In communicated systems, the power reference and the power share of the generators is known, being a useful information for improving the disturbance rejection of the controller. As an alternative, in communication-less solutions, derivative action is commonly used to fasten the response by increasing the controller phase margin. However, it is affected by noise and oscillations on the estimated frequency signal. 3) The grid frequency reference, which may vary governed by droops in the grid, is unknown; leading to power share during steady state if the reference used in the compensation does not match the frequency command.

The present paper will focus on the design of an improved transient frequency drift compensator. For that purpose, the existing methods are analyzed and some enhancements are proposed concerning the commented problems. Among the contributions, it is proposed the use of a Luenberger type observer [12] for the estimation of the load current and the reduction of the compensator phase lag due to the grid frequency estimator.

The paper is organized as follows. Section II formulates the dynamic frequency drift problem. Section III covers the proposed control algorithms and the existing limitations. Section IV details the use of the Luenberger based observer for load 
estimation. Finally, the experimental results are shown at Section V.

\section{PRoblem Definition AND SYSTEM MODELING}

Deviations on power grid nominal parameters can lead to non-optimal operation or malfunction of grid connected elements. Moreover, the violation of regulations could trigger the system protections, compromising the grid power quality and stability. As a reference, the default IEEE regulation for Distributed Resources is summarized in Table I.

TABLE I

DEFAULT CLEARING TIMES UNDER ABNORMAL VOLTAGE AND FREQUENCY OPERATION BASED ON IEEE STD 1547A-2014 [13]

\begin{tabular}{c|c}
\hline Voltage range $(\%$ of base voltage) & Clearing Time (s) \\
\hline $\mathrm{V}<45$ & 0.16 \\
$45 \leq \mathrm{V}<45$ & 1 \\
$60 \leq \mathrm{V}<88$ & 2 \\
$110 \leq \mathrm{V}<120$ & 1 \\
$\mathrm{~V} \geq 120$ & 0.16 \\
\hline Frequency(Hz) & Clearing Time (s) \\
\hline$f_{n}<f_{n}-3$ & 0.16 \\
$f_{n}<f_{n}-0.5$ & 2 \\
$f_{n}>f_{n}+0.5$ & 2 \\
$f_{n}>f_{n}+2$ & 0.16 \\
\hline
\end{tabular}

MGs and Distribution Networks are often governed by low inertia synchronous generators, being the grid active power related to their rotating speed. On weak grids, the connection and disconnection of the different elements presented in the grid as power generators and loads may cause variations on the voltage magnitude and frequency. This problem, illustrated in Fig. 1, can be mitigated by using a solution capable of injecting power to the grid with a much faster dynamic response than the grid generators, compensating the power mismatch.

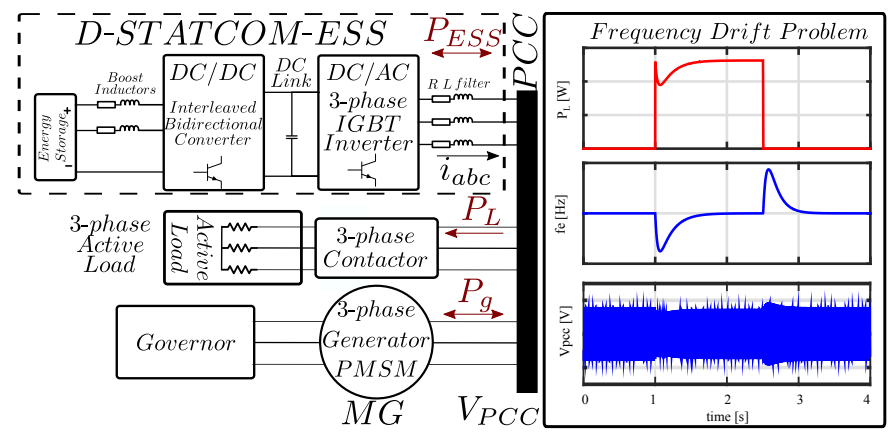

Fig. 1. Integration of the system under study in a simplified MG model for experimental purpose. The frequency drift contingency is illustrated as a graph showing the load power and the consequent grid frequency disturbance.

Fig. 1 shows the proposed solution integrated in an experimental simplified 1 node set-up, emulating a simplified MG, composed by a synchronous generator acting as the main supply and a pure active 3-phase load. The compensation solution will be based on an autonomous system constituted by a D-STATCOM, consisting on a 3-phase 2-level IGBT inverter, coupled through a DC link to an ESS formed by a battery, or any other bidirectional DC supply, and a bidirectional DC/DC boost IGBT converter in 2 branch interleaved configuration. The figure shows the power flow in the system, being $P_{g}$, $P_{L}$ and $P_{E S S}$ the generator, the load and the proposed solution power respectively. The proposed topology will be able to both inject or consume power, thus, it will assist the generator sharing active power only during transients due to load connections and disconnections, smoothing the frequency dynamic response. On the other hand, during steady state operation, the ESS should be idle unless the battery needs to be charged.

\section{A. Generator model}

For an optimal frequency compensation, the system dynamic model must be known. Moreover, the system modeling becomes mandatory when observation techniques are used. Fig. 2 shows the equivalent system of a synchronous generator coupled to a governor which controls the shaft speed with a certain speed control loop. In the figure, $\omega_{r}$ is the rotor mechanical speed in rad/s, $C_{g}$ is the governor controller, $T_{g}$ is the torque injected by the governor, $J$ and $b$ are the generator mechanical parameters (inertia and friction coefficient), $K_{e}$ and $K_{t}$ are the generator back EMF and torque constant and $p$ is the number of pole pairs. The electrical load is represented by the parameters $R_{T}$ and $L_{T}$ being $i_{L}$ the load current and $T_{L}$ the equivalent torque load. $T_{E S S}$ is the equivalent torque of the proposed compensating solution. $R_{g}$ and $L_{g}$ represent the grid impedance, in this case the generator stator impedance.

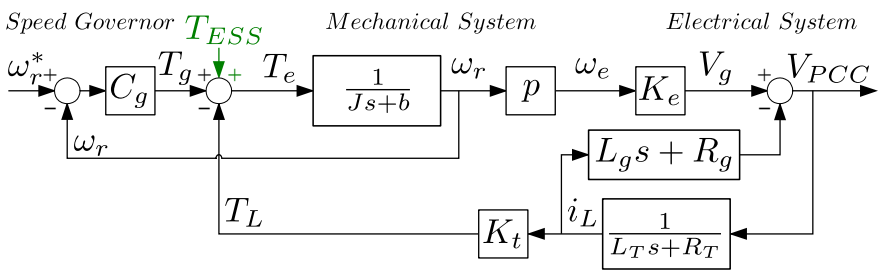

Fig. 2. Equivalent system diagram of a PM synchronous generator supplying constant-impedance type loads.

The mechanical system of the generator its coupled to its electrical one, thus any change or disturbance in the electrical grid will be reflected in its mechanical system. In the case an electrical active load, $R_{T}$, is suddenly connected to the grid, it will generate an equivalent torque load $T_{L}$ proportional to the load current $i_{L}$ at the generator's shaft. This torque, will be seen as a disturbance by the governor, causing a speed variation which depends on the mechanical inertia and the governor control system. Considering the control system of governor as a PI in the ideal form and neglecting the effect of electrical subsystem of the generator, the generator disturbance transfer function $D_{g}$ is expressed by (1).

$$
D_{g_{(s)}}=-\frac{\omega_{r(s)}}{T_{L(s)}}=\frac{1}{C_{g(s)}+J s+b}=\frac{\frac{1}{J} s}{s^{2}+\frac{K_{p}+b}{J} s+\frac{K_{p}}{T_{i} J}}
$$

Attending to the generator system, it is worth noting that the generator voltage is proportional to the mechanical speed 
through the back electromotive force constant $\left(K_{e}\right)$. Thus, as well as the frequency, the voltage magnitude will variate proportionally. Fig. 1 illustrates the behavior of the synchronous generator when a load is connected/disconnected. As it can be seen, both the frequency and the voltage are affected. Therefore, depending on the grid $X / R$ ratio, the compensation could positively affect the active and reactive power.

\section{TRANSIENT FREQUENCy DRIFT COMPENSATION}

The basic idea of the dynamic frequency drift compensation consists on maintaining the active power balance between the grid elements during transients. In the case under study, the transient active power mismatch between the generation and consumption in the grid will depend on the grid equivalent inertia determined by the synchronous generators and their associated power source characteristics. Thus, one of the first ideas that emerged consisted on the modification of such grid inertia by introducing a virtual inertia using an ESS that emulates the behavior of a synchronous generator, known in the literature as Virtual Synchronous Machine (VSM) [8]. Although in the literature the expression is commonly referred as a relation between injected power and frequency, in this case an expression in terms of torque and frequency seems to be more appropriate. The law of control is summarized in (2)

$$
T_{E S S(t)}=k_{p E S S}\left(w_{e(t)}^{*}-w_{e(t)}\right)-J_{E S S} \cdot s \cdot w_{e(t)}
$$

where $T_{E S S}$ is the equivalent torque, $k_{p E S S}$ is a damping coefficient that emulates a governor with proportional regulator, $J_{E S S}$ is the virtual inertia gain that emulates a machine inertia, $w_{e}$ is the instantaneous grid frequency in rad/s and $w_{e}^{*}$ the frequency reference. At this point it is worth to notice that for the case of a single generator grid at which all the machine parameters are known and the frequency is perfectly measured, the term related to the inertia $J_{E S S} \cdot s \cdot w_{e(s)}$ would be sufficient to completely compensate the transient response. However, in a real situation, and considering no communications are used, the parameters uncertainty, changes at the operating conditions as well as limited sensor bandwidth and digital control delay, make necessary the use of techniques to improve the control for frequency compensation. The possible enhancements go from the use of a proportional term $k_{p E S S}$ used in VSGs, to advanced techniques as the ones proposed in [8]. The present study, covers improvements based on the use of of the available system information in order to estimate the unknown parameters and to enhance the overall performance, taking special concern on the frequency sensor effects. The conditions and constraints of the proposed communication-less solution are the following:

- There is not communication between the proposed solution and any other system at the grid.

- The available measurements will consist only on electric variables (currents and voltages) at the PCC.

- The control relays on the feedback of grid frequency, thus, frequency estimation methods are needed.
- The power exchange between the proposed system and the grid should take place only during transients. The charge cycles of the ESS are not covered in this study.

- Actual frequency command in the MG is unknown.

- The possibility of binding the proposed solution to a significant load in the grid, gaining access to the load current is also considered.

Under these constraints, the main problems to be tackled are summarized as:

- The frequency has to be estimated from electrical variables. The effects of measurement noise and delays as well as grid distortion over the used frequency estimation techniques must be considered.

- The transient state should be detected in order to avoid any possible power share during steady state.

- The frequency command is unknown while is one of the variables involved. Its effects must be either estimated or decoupled.

- The digital implementation of the control introduces constraints relative to delay, bandwidth limitation and noise that can affect the performance being specially critical the use of derivatives.

\section{A. Proposed Control System}

A simplified version of the proposed control system scheme is shown in Fig. 3. Besides the internal active and reactive current control loops of the converter, five blocks can be identified forming the communication-less solution.

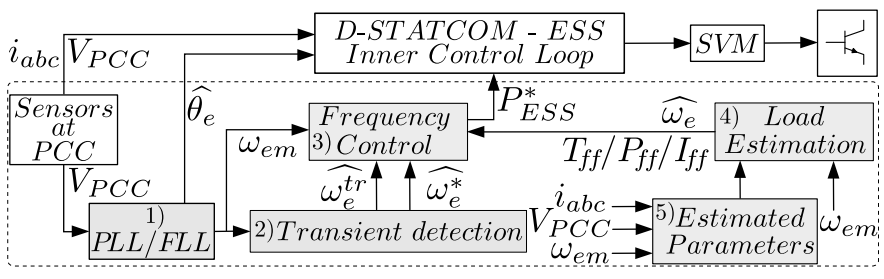

Fig. 3. Simplified control scheme for the proposed system.

1) Grid synchronization and frequency estimation: Ir provides the estimated grid angle $\left(\widehat{\theta}_{e}\right)$ for the current coordinate transformation, and the estimated grid frequency $\left(\widehat{\omega}_{e}\right)$ for its use as control feedback signal, based on PLL or FLL techniques. An experimental comparison of both PLL and FLL frequency measurements with respect to the reading giving by an observer is shown at Fig. 4. As it is readily seen, both PLL with low pass filter and FLL are good candidates for the frequency estimation. However, considering the robustness of FLL under distorted grids, this last solution will be used on all the subsequent experiments.

2) Transient detection: As the power exchange during steady state should be avoided, the detection of frequency transients is required. Moreover, the actual grid command should be decoupled from the transient in order to not to react to variations of the frequency grid reference. The proposed method is detailed at section III-B. 


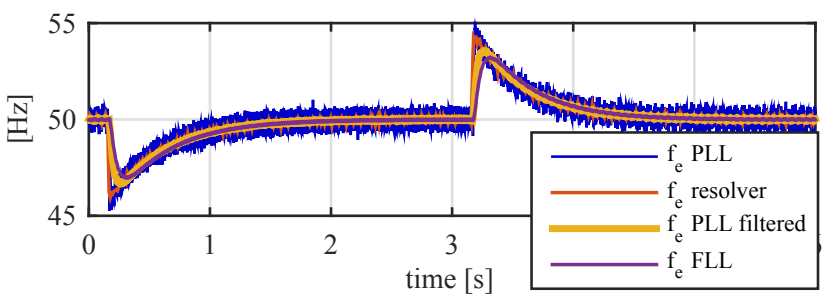

Fig. 4. Experimental results for the measurement of the frequency drift. As a reference the signals are compared with the one given by a resolver. For this work, an FLL is considered.

3) Frequency control: The main block of the control system consist on a regulator able to provide a power reference for the D-STATCOM $\left(P_{E S S}^{*}\right)$, using the error between the frequency reference and the feedback frequency estimation. Details for the control tuning are giving at section III-C.

4) Load disturbance estimation: When grid parameters are known or estimated, it is possible to generate a load estimation and use it as a feedforward either in terms of power, torque or $\operatorname{current}\left(P_{F F}, T_{F F}, I_{F F}\right)$ to improve the response. In this study two options have been contemplated one based on current measurement, developed at section III-D, and another relaying on a Luenberger-based observer, considered at section IV.

5) Estimated parameters: The necessary information for the implementation of the load disturbance estimator should be estimated either online or offline. In the scope of this paper all the parameters will be known or in any case offline obtained.

\section{B. Transient detection}

As it has been said before, it is needed to detect a frequency change with respect to the reference. With the proposed communication-less approach, there is no possibility of getting the grid frequency reference and the detection must be completely carried out autonomously. The obvious solution is to apply a high-pass filter and, over the resulting signal, to calculate the absolute value, which compared with a threshold level will be used for triggering the power injection. However, those kind of methods will still need to subtract from the measured grid frequency the estimated reference. For this reason, the method is prone to errors due to small variations on the reference frequency.

In this paper, a method based on the correlation of the measured frequency with a signal of period $T$ and zero average is proposed (3).

$$
u(t)=\left(\int_{0}^{T} x(t) f(t) d t\right)^{2}
$$

where $x(t)$ is $\hat{\omega}_{e}$ and $f(t)$ could be a sine or cosine.

The correlation is digitally calculated in two steps: 1) At each sample time, the value for the inner integral is calculated and the result is stored in a variable in memory

$$
C \omega_{e[k]}=C \omega_{e[k-1]}+\widehat{\omega}_{e[k]} \cdot \sin (2 \pi k T)
$$

2) After completing the samples for one correlation signal period, the square of the integral is calculated and the result saved:

$$
C^{2} \omega_{e[k]}=\left(C \omega_{e[k]}\right)^{2}
$$

Following, the last calculated value for the $C^{2} \omega_{e[k]}$ variable is compared with a selected threshold. In the case the value is smaller than the threshold, meaning the system is at steady state, the reference frequency is updated to the measured one. When the value exceeds the threshold, the transient condition is activated and the reference frequency hold to the last updated value. Finally, the estimated transient frequency is obtained as

$$
\widehat{\omega}_{e}^{t r}[k]=\widehat{\omega_{e}}[k]-\widehat{\omega_{e}^{*}}[k]
$$

The obtained experimental results for the estimated transient frequency are shown at Fig. 5. The results are compared with

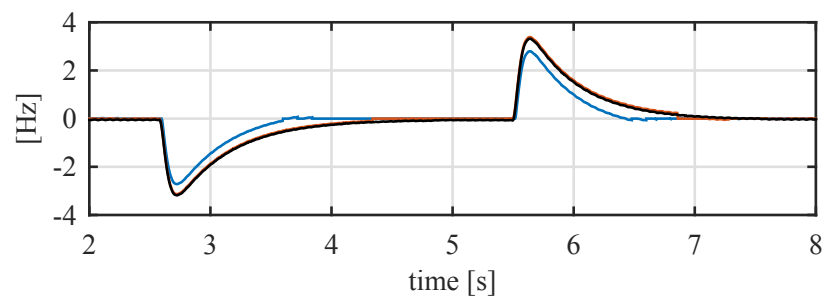

Fig. 5. Experimental results for the transient frequency estimation using the correlation method. The FLL estimated frequency with the reference value subtracted is shown in black. The estimated transient is shown using two different values for the sinusoidal signal period, blue curve is for $T=40 \mathrm{~ms}$, red one for $T=70 \mathrm{~ms}$. Threshold value is set for both cases to 5 .

respect to the measured frequency, from which the reference value has been subtracted. The curves are presented using two different values for the period of the base sinusoidal signal. As it can be seen, the selection of the period greatly affect the accuracy of the method. Still, the results are really promising while keeping the computational burden very low.

\section{Frequency feedback based control}

The basic idea for the feedback based control is shown at Fig. 6. The feedback frequency controller, $C_{c}$, uses the estimated frequency reference $\left(\widehat{\omega}_{e}^{*}\right)$ and the measured value $\left(\widehat{\omega}_{e}\right)$ to provide a control action that could be interpreted either as a power $\left(P_{E S S}^{*}\right)$ or equivalent torque command $\left(T_{E S S}^{*}\right)$. In this paper, the use of the torque command is proposed.

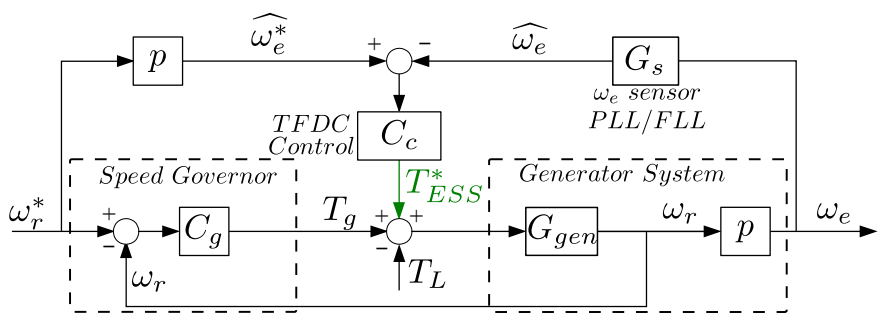

Fig. 6. Block diagram of the transient frequency drift compensator based on feedback control. The provided torque reference is highlighted in green.

The analysis of the dynamic performance of the feedback system it is based on the the disturbance rejection transfer functions. As it has been explained before, for the governor 
system without any external compensation, the $T_{L}$ to $\omega_{r}$ transfer function is given by the expression of $D_{g_{(s)}}(1)$. When the external compensation is added, the resulting transfer function is given by (7)

$$
D_{c(s)}=\frac{\omega_{r}}{T_{L}}=-\frac{D_{g(s)}}{1+D_{g(s)} \cdot C_{c(s)} \cdot G_{s(s)}^{\prime}}
$$

where $G_{s(s)}^{\prime}=G_{s(s)} \cdot p$, being $p$ the number of pole pairs of the machine. For the analysis, the generator disturbance transfer function and the frequency sensor are approximated by second order transfer functions as shown in (8). These expressions will be also later used during the development of the observerbased compensation.

$D_{g(s)}=\frac{k_{g} \omega_{n g}^{2} s}{s^{2}+2 \xi_{g} \omega_{n g} s+\omega_{n g}^{2}}, G_{s(s)}=\frac{k_{s} \omega_{n s}^{2}}{s^{2}+2 \xi_{s} \omega_{n s} s+\omega_{n s}^{2}}$

where the gain $k_{g}$ is the $D_{g}$ gain, and $k_{s}$ is the sensor gain, 1 by default, while $\omega$ and $\xi$ are the natural frequency and damping factor of each system. If the governor is considered as a PI regulator, the parameters are equivalent to $k_{g}=\frac{T_{i}}{K_{p}}$, $\omega_{n g}=\sqrt{\frac{K_{p}}{T_{i} J}}$ and $\xi_{n g}=\frac{b+K_{p}}{2 J \omega_{n g}}$.

Fig. 7 shows the general scheme for the frequency controller $C_{c}$, including the torque and power alternatives. The $R$ and $H$ functions can be substituted by the common regulators terms. P, PI, PD and PDF (pseudo-derivative feedback [14]). It is highlighted in the figure the possibility for adding a feedforward to improve the control. Detailed explanation about those modifications will be shown in III-D. where $u(s)$ is the

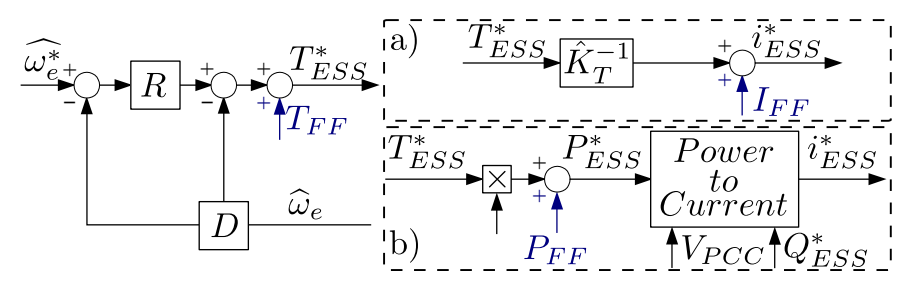

Fig. 7. Control structure for frequency drift compensation. R could be a $\mathrm{P}$, PI or PD regulator and $\mathrm{D}$ is 0 or derivative regulator (PDF). a) Torque alternative, b) Power alternative.

control action $\left(T_{E S S}^{*}\right)$ and $e(s)$ the error $\left(\omega_{e(s)}^{*}-\omega_{e(s)}\right)$.

Before starting with the evaluation of each method, it is worth noting that PI and PD methods are less appealing for this application. The use of an integral action in the controller, even if it can improve the disturbance rejection drastically, would lead to the unavoidable share of power during steady state. On the other hand, the inclusion of a differential action in the direct chain, will boost noise problems in the case there are changes at the commanded frequency. Moreover, PDF topology not only offers derivative action in an smarter way than a classical PD but also, as seen before, has a physical meaning and has been used before in similar frequency control applications [8]. Even being a limited option, $\mathrm{P}$ regulator appears as a simpler solution that might be enough for some applications.
The method selected for the comparison of the feedback alternatives is based on the evaluation of the dynamic stiffness. Being the proposed control system oriented to the disturbance rejection, the method will provide a clear view on the expected performance. For the analyzed problem, the dynamic stiffness is defined as (9)

$$
S_{c(s)}=\frac{T_{L}}{\omega_{r}}=\frac{1+D_{g_{(s)}} \cdot C_{c(s)} \cdot G_{s(s)}^{\prime}}{D_{g_{(s)}}}
$$

When the dynamic stiffness transfer function is evaluated in frequency domain for different $\mathrm{P}$ and PDF settings, the results shown in 8 are obtained. The graph clearly indicates the benefits of an increased proportional gain and also the additional disturbance rejection capability bandwidth because of the inclusion of the differential term. The corresponding transient responses in time domain for the same design cases shown at Fig. 8, are depicted at Fig. 9. As shown, the predicted benefits for including the differential term are translated to a reduced initial overshoot when compared to the $\mathrm{P}$ design.

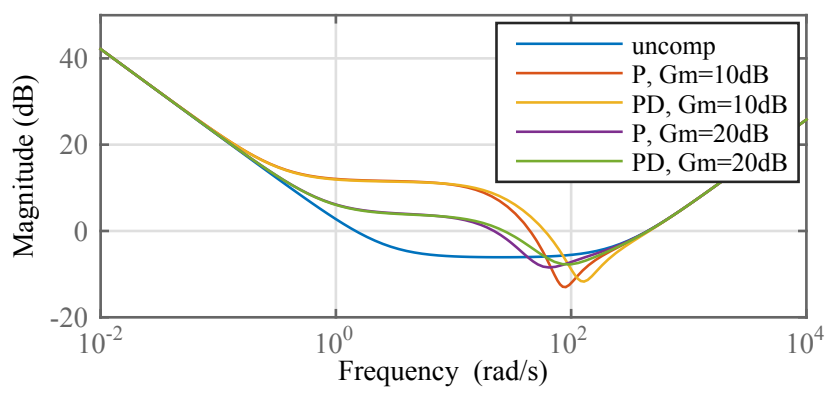

Fig. 8. Dynamic stiffness for PD and PDF methods with two different proportional gains. For the case of PDF methods, a minimum phase margin of $40 \mathrm{deg}$. is set at the open loop crossover frequency.

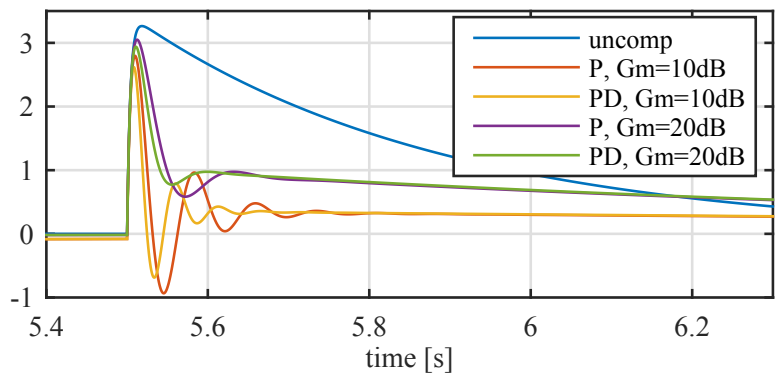

Fig. 9. Compared transient responses for PD and PDF methods with two different proportional gains. For the case of PDF methods, a minimum phase margin of $60 \mathrm{deg}$.

Finally, the experimental results for the feedback-based compensation is shown at Fig. 10. A good agreement with respect to the previous theoretical discussion can be observed.

D. Improving the basic control: Load feed-forward and state observation

Given the limitations due to phase lag on the frequency estimation algorithm, some solutions could be applied to increase the phase and gain margins, improving the response of 
the dynamic frequency controller. Two ideas are proposed, the use of a load disturbance feed-forward and the compensation of the frequency estimation effect.

If the load information is available either through load current measurement, or any other method, it can be used as a feed-forward for the controller, as shown in Fig. 7, considering the load feed-forward either in terms of current, power or torque. Ideally, if the load is known and it is fully compensated by the ESS, the frequency drift will be eliminated. However that solution would inject the whole load power during steady state. In order to compensate only the transient and keep the grid frequency unalterable, the ESS has to share only the power load minus the power drawn by the governor, i.e. the equivalent torque of the compensation system should be $T_{E S S}=T_{L}-T_{g}$ in order to reach an optimal balance between compensation and injected energy. The relation between $T_{g}$ and $T_{L}$ is written in (10) being the transfer function for the ideal case $\frac{T_{E S S}}{T_{L}}=1-\frac{T_{g(s)}}{T_{L(s)}}$.

$$
\frac{T_{g_{(s)}}}{T_{L(s)}}=\frac{C_{g_{(s)}}}{C_{g_{(s)}}+J s+b}=\frac{K_{p} s+K_{p} / T_{i}}{J s^{2}+\left(K_{p}+b\right) s+K_{p} / T_{i}}
$$

Nevertheless, unlike $D_{g_{(s)}}$, obtaining $\frac{T_{g_{(s)}}}{T_{L(s)}}$ is not easy in a communication-less system if the system parameters are unknown. However, the load information is still a valuable information as it is a derivative state of the frequency, allowing to anticipate the control reaction, i.e. to reduce the phase delay gaining phase margin. In order to eliminate the share during steady state instead of TF in (10), a high pass filter(HPF) can be applied to the load information.

Fig. 10 shows the experimental performance comparison of the different feedback methods and the response when a load feed-forward is used. The load information comes from a load current sensor, which is a valid assumption considering the the proposed solution is bind to a significant load. The full load compensation is also shown. Is has been obtained by equaling the D-STATCOM current reference to the load measured current.
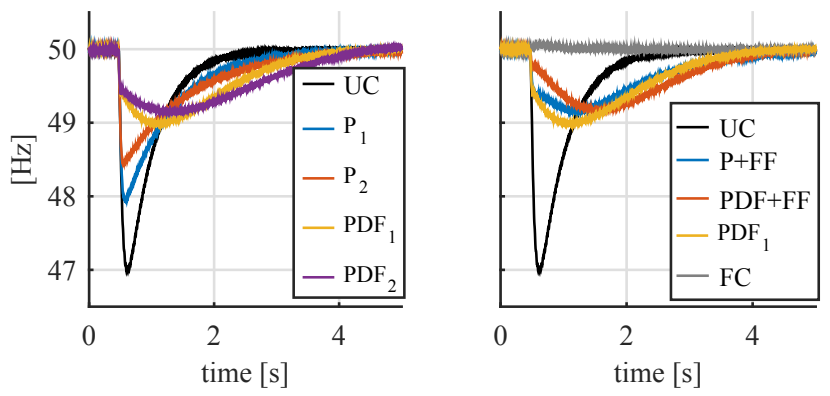

Fig. 10. Transient frequency drift compensation using different control methods. a) Grid frequency, b) Injected Power. UC: uncompensated, $P_{1}$ : P controller, $K_{p}=0.1, P_{2}: \mathrm{P}$ controller, $K_{p}=0.2, P D F_{1}$ : PDF controller, $K_{p}=0.2, K_{d}=0.1, P D F_{2}$ : PDF controller, $K_{p}=0.2, K_{d}=0.25$, $P D F+F F:$ PDF with load feedforward, 2nd order high-pass filter, bandwidth $=0.5 \mathrm{~Hz}$, and same gains as $P D F_{1}$.

\section{LOAD OBSERVER MODELLING}

The proposed Luenberger-based observer control is shown in Fig. 11. In this scheme, the speed governor and the generator will be considered as the system and the $P L L / F L L$ the sensor. Considering that the generator and governor param-

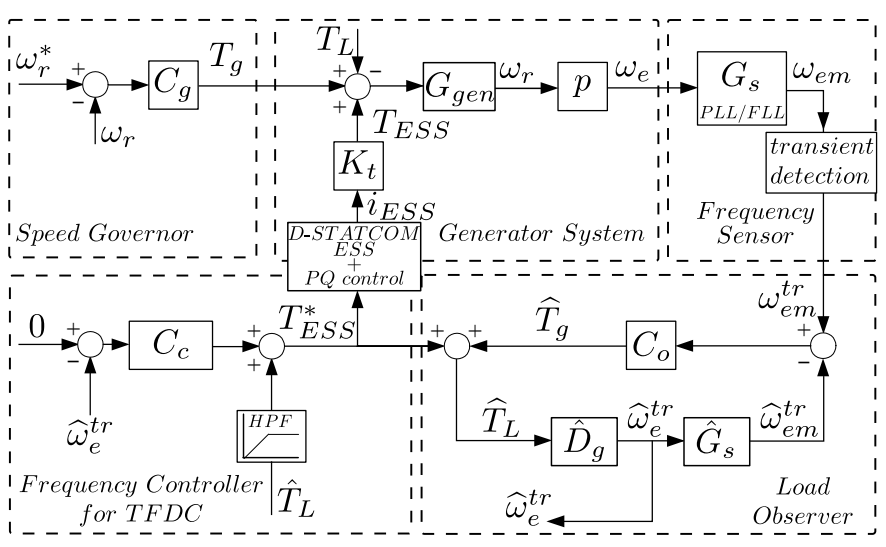

Fig. 11. Proposed observer control structure .

eters are unknown, the systems $C_{g}$ and $G_{g e n}$ can not be used explicitly in the proposed solution. However, the second order approximations proposed before for both the sensor and the generator disturbance transfer functions (8), are appealing candidates to the observer implementation. The experimental comparison for the proposed sensor and generator disturbance transfer functions with respect to the real systems are shown in Fig. 12. For the observer implementation, state space
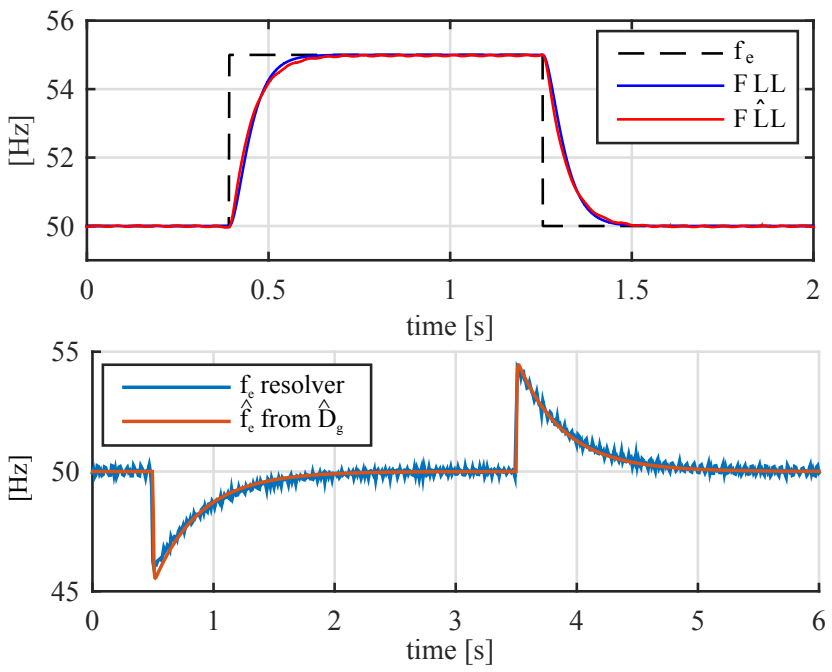

Fig. 12. Experimental results. Top, DSOGI FLL response and its approximation using the $2^{\text {nd }}$ order TF in (8). Bottom, Open-loop response obtained with the estimation of the disturbance TF $\hat{D}_{g}$. The estimated frequency is compared with the value obtained by the generator resolver.

modeling is considered. The observer plant state space model is shown in Fig. 13. The model is defined by the state vector $x=\left[x_{1}, x_{2}, x_{3}, x_{4}\right]^{T}$, the input vector $u=\left[T_{L}\right]$ and the output vector $y=\left[\omega_{e}, \omega_{e m}\right]^{T}=\left[p \cdot x_{1}, x_{3}\right]^{T}$. The state, input, output 


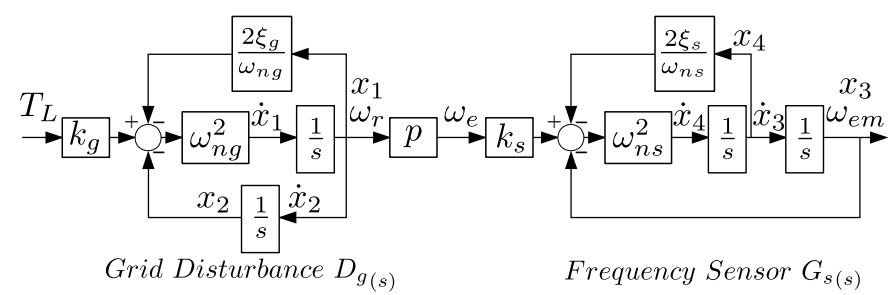

Fig. 13. Observer system plant block diagram in state space form.

and feedforward matrices are defined in (11).

$$
\begin{gathered}
A=\left[\begin{array}{cccc}
-2 \xi_{g} \omega_{n g} & -\omega_{n g}^{2} & 0 & 0 \\
1 & 0 & 0 & 0 \\
0 & 0 & 0 & 1 \\
k_{s} p \omega_{n s}^{2} & 0 & -\omega_{n s}^{2} & -2 \xi_{s} \omega_{n s}
\end{array}\right], B=\left[\begin{array}{c}
k_{g} \omega_{n g}^{2} \\
0 \\
0 \\
0
\end{array}\right] \\
C=\left[\begin{array}{cccc}
p & 0 & 0 & 0 \\
0 & 0 & 1 & 0
\end{array}\right]_{[k]}, \quad y_{[k]}=C_{d} x_{[k]}+\left[\begin{array}{c}
0 \\
0
\end{array} D_{d} u_{[k]}\right.
\end{gathered}
$$

For the digital implementation the difference equations in (12) are used, where $A_{d}, B_{d}, C_{d}$ and $D_{d}$ are the discretized versions of the state space matrices using zero order hold $(\mathrm{ZOH})$. The discrete matrices are obtained as $A_{d}=e^{A T_{s}}$, $B_{d}=A^{-1}\left(A_{d}-I\right) B, C_{d}=C$ and $D_{d}=D$ being $T_{s}$ the sampling time and $I$ the identity matrix of $A_{d}$. The values used for the listed parameters are shown in Table II.

For the observer analysis, as previously done for the feedback-based control, the dynamic stiffness is used. From the proposed observer block diagram, and assuming parameters matching between the real system and the observer equations, (13) can be obtained.

$$
\frac{T_{L}}{\omega_{e}}=\frac{1+\widehat{D_{g}} \cdot C_{o} \cdot \widehat{G_{s}}}{1+\widehat{D_{g}}\left(C_{c}+C_{o} \cdot \widehat{G_{s}}\right)}
$$

The resulting frequency plots and transient response is shown in Fig. 14 and Fig. 15. The curves are compared with the uncompensated, ideal sensor and feedback compensation. As it can be seen, the use of the observer improves in a noticeable way the initial transient response due to the compensated sensor lag. Tuning of the observer has been done using loop shaping techniques in frequency domain. The order of the controller has been kept equal to the number of poles of $\widehat{D_{g}} \cdot \widehat{G_{s}},\left(4^{t h}\right)$. Values for the observer feedback controller are listed in Table II.

\section{EXPERIMENTAL RESULTS}

The proposed solutions has been tested both simulated and experimentally, following the setup presented in Section II and in Fig. 1, and the parameters in Table II. In the experimental setup, for the implementation of the DC/DC converter and the inverter, two MTL-CBI0060F12IXHF power converters rated at $25 \mathrm{~kW}$ have been used. The control system has been implemented in a TMS320F28335 DSC from Texas Instruments. The MG generator is emulated using 2 coupled PMSMs one acting as generator and other as governor motor controlled by a commercial drive.

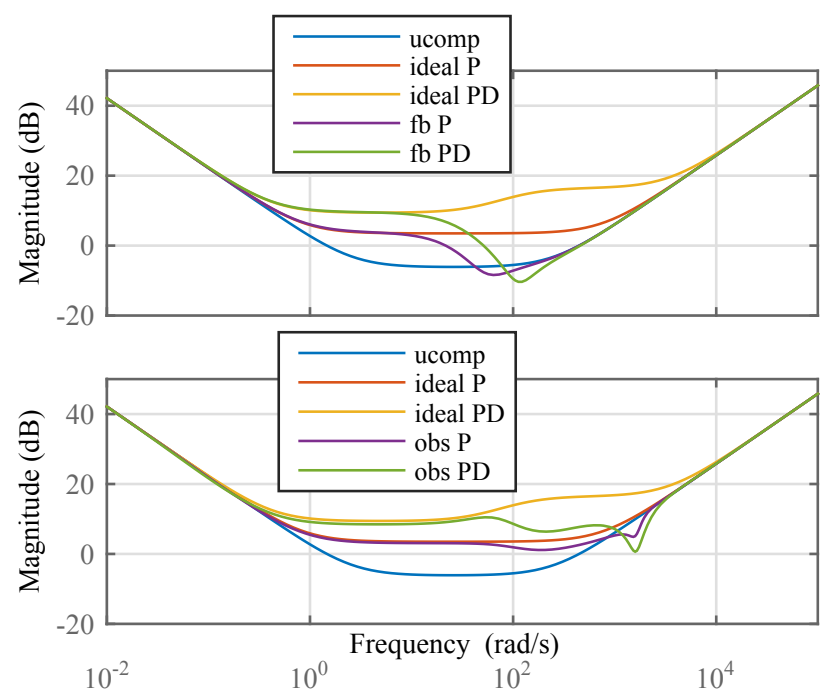

Fig. 14. Dynamic stiffness. Top axes, results for the feedback-based control. Bottom axes, observer-based control. The uncompensated and ideal sensor cases are shown as a reference.

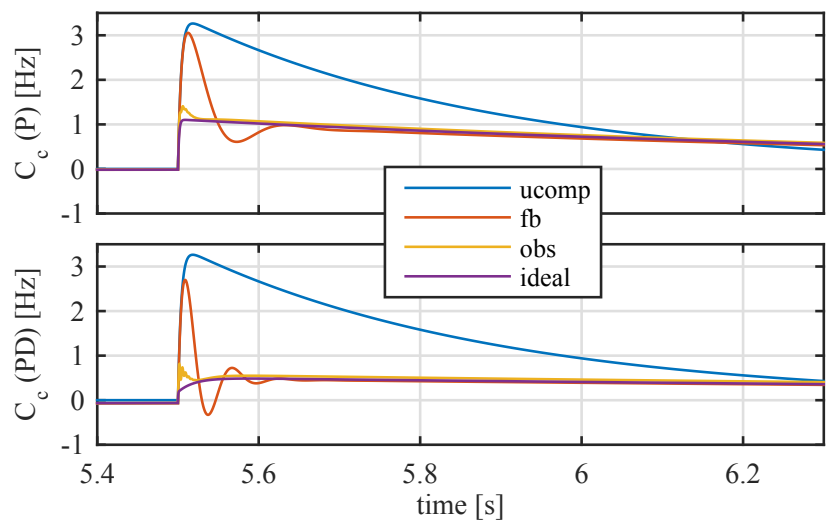

Fig. 15. Transient response. Top axes, results when a $\mathrm{P}$ controller is used as the transient frequency controller $C_{c}$. Bottom axes, results when a PD is used instead. The uncompensated and ideal sensor cases are shown as a reference.

The grid currents and voltages are shown at Fig. 16, a comparison for the feedback and feedforward methods at Fig. 17 and the observer based results compared with respect to the other analyzed method as well as the estimated load are shown in Fig. 18 and Fig. 19. As expected, feedforward and observer based methods have the best performance in terms of frequency compensation. The use of observation techniques also allows to estimate the load current.

\section{CONCLUSIONS}

In this paper, an autonomous system has been proposed for transient frequency drift compensation. The proposed solution has addressed some of the limitations of existing literature. The performance achieved by feedback control has been improved by the employment of feedforward current and the development of a Luenberger-based observer. A detailed comparison including analytical derivations, simulation and experimental results have been carried out. The proposed signal processing 
TABLE II

SYSTEM PARAMETERS

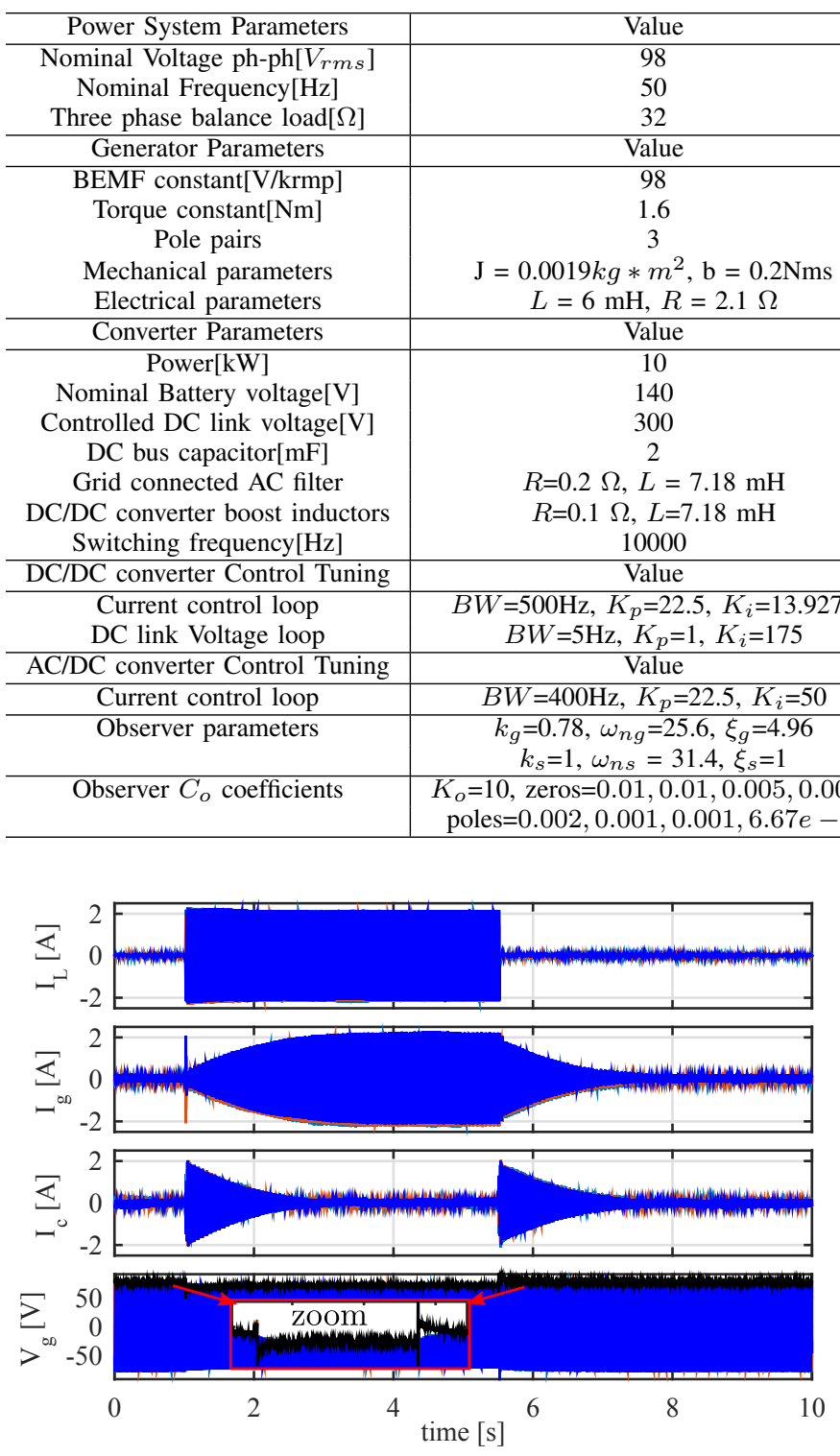

Fig. 16. Experimental results showing the current and voltages. From top to bottom, load current, generator current, compensation current and grid voltage.

for removing of the steady state frequency reference, based on the use of a correlation signal, enables the method to work on weak grids operated under droop control.

Finally, a comparative evaluation of the analyzed techniques has been done summarizing their performance in Fig. 20. Five indexes are considered. The maximum frequency deviation $\Delta f_{\max }$, the peak power $\left(P_{\max }\right)$, the total shared energy $(E)$ and two coefficients $C_{\text {comp }}$ and $C_{\text {sett }}$ are defined to evaluate the initial compensation and the enlargement of settling time compared to the original response respectively. The indexes are shown normalized in pu, being all of them the higher the worst, except $C_{c o m p}$ that represent an advantage when tends towards one.
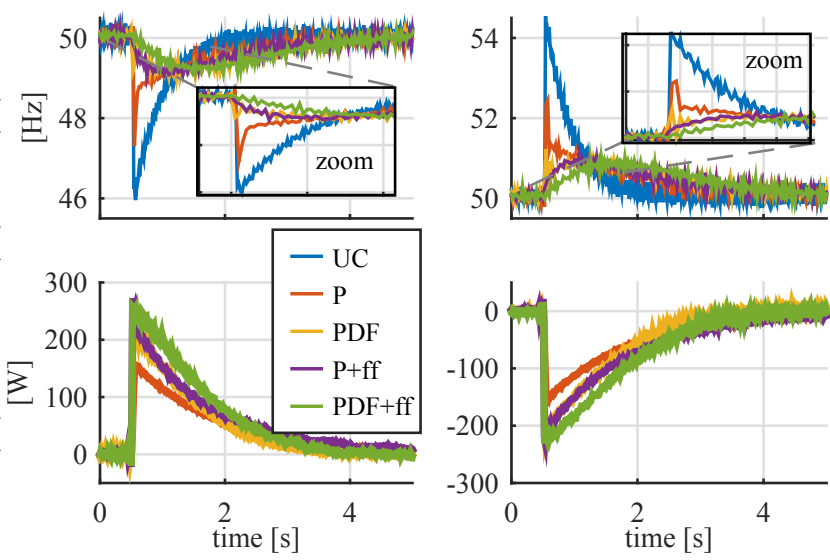

Fig. 17. Experimental results. Grid frequency and Injected Power. UC: uncompensated, $P: \mathrm{P}$ controller, $K_{p}=0.2, P D F$ : PDF controller, $K_{p}=$ $0.2, K_{d}=0.1, P+F F$ and $P D F+F F:$ P, PDF controllers with load feedforward 1st order high-pass filter, $\mathrm{bw}=0.5 \mathrm{~Hz}$, and same gains as $P$ and $P D F$.

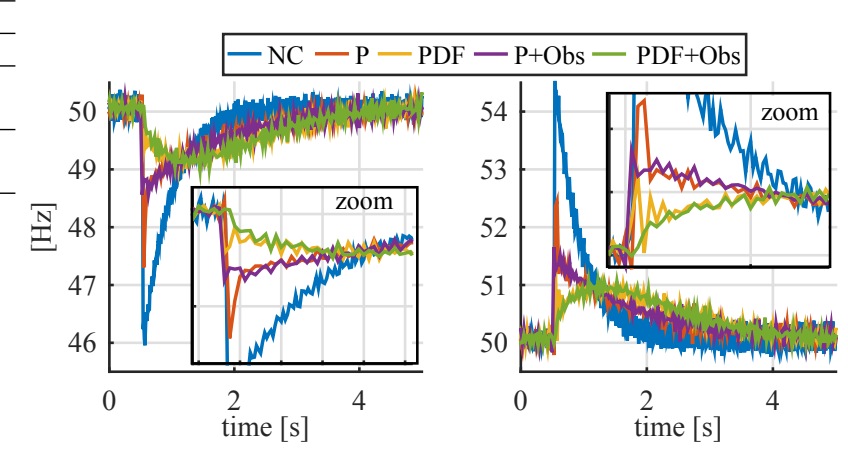

Fig. 18. Experimental results. Observer results. UC: uncompensated, $P$ and $P D F$ same than at Fig. 16. $P+O b s, P D F+O b s$ observer bases estimation with the parameters shown at Table II.

\section{REFERENCES}

[1] J. M. Guerrero, "Editorial special issue on power electronics for microgrids 2014;part ii," IEEE Transactions on Power Electronics, vol. 26, no. 3, pp. 659-663, March 2011.

[2] J. Guerrero, P. C. Loh, T.-L. Lee, and M. Chandorkar, "Advanced control architectures for intelligent microgrids 2014;part ii: Power quality, energy storage, and ac/dc microgrids," IEEE Transactions on Industrial Electronics, vol. 60, no. 4, pp. 1263-1270, April 2013.

[3] A. Agbedahunsi, M. Sumner, E. Christopher, A. Watson, A. Costabeber, and R. Parashar, "Frequency control improvement within a microgrid, using enhanced statcom with energy storage," in 6th IET International Conference on Power Electronics, Machines and Drives (PEMD 2012), March 2012, pp. 1-6.

[4] A. Arulampalam, J. Ekanayake, and N. Jenkins, "Application study of a statcom with energy storage," IEEE Proceedings on Generation, Transmission and Distribution, vol. 150, no. 3, pp. 373-384, May 2003.

[5] M. Falvo, L. Martirano, and D. Sbordone, "D-statcom with energy storage system for application in smart micro-grids," in 2013 International Conference on Clean Electrical Power (ICCEP), June 2013, pp. 571576.

[6] M. Holmberg, M. Lahtinen, J. McDowall, and T. Larsson, "Svc light with energy storage for frequency regulation," in 2010 IEEE Conference on Innovative Technologies for an Efficient and Reliable Electricity Supply (CITRES), Sept 2010, pp. 317-324.

[7] I. Serban and C. Marinescu, "Control strategy of three-phase battery energy storage systems for frequency support in microgrids and with uninterrupted supply of local loads," IEEE Transactions on Power Electronics, vol. 29, no. 9, pp. 5010-5020, Sept 2014. 


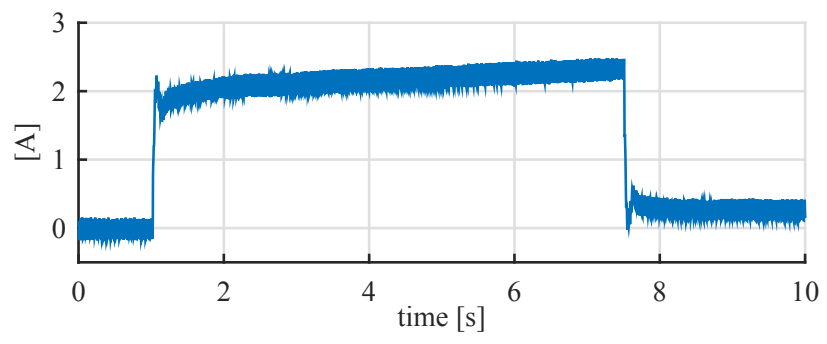

Fig. 19. Experimental results. Observer estimated load. The real current load was $2.2 \mathrm{~A}$.

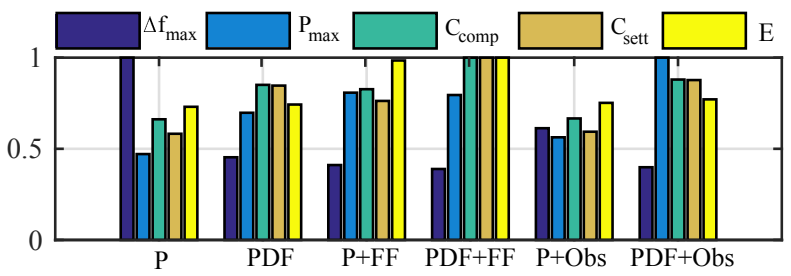

Fig. 20. Merit figure. Performance evaluation for all the considered methods.

[8] M. Torres L, L. Lopes, L. Moran T, and J. Espinoza C, "Self-tuning virtual synchronous machine: A control strategy for energy storage systems to support dynamic frequency control," Energy Conversion, IEEE Transactions on, vol. 29, no. 4, pp. 833-840, Dec 2014.

[9] S.-K. Chung, "A phase tracking system for three phase utility interface inverters," IEEE Transactions on Power Electronics, vol. 15, no. 3, pp. 431-438, May 2000.

[10] P. Roncero-Sanchez, X. del Toro Garcia, A. Torres, and V. Feliu, "Fundamental positive- and negative-sequence estimator for grid synchronization under highly disturbed operating conditions," IEEE Transactions on Power Electronics, vol. 28, no. 8, pp. 3733-3746, Aug 2013.

[11] P. Rodriguez, A. Luna, M. Ciobotaru, R. Teodorescu, and F. Blaabjerg, "Advanced grid synchronization system for power converters under unbalanced and distorted operating conditions," in 32nd Annual Conference on IEEE Industrial Electronics, IECON 2006, Nov 2006, pp. 5173-5178.

[12] D. Luenberger, "Observing the state of a linear system," IEEE Transactions on Military Electronics, vol. 8, no. 2, pp. 74-80, April 1964.

[13] "Ieee standard for interconnecting distributed resources with electric power systems - amendment 1," IEEE Std 1547a-2014 (Amendment to IEEE Std 1547-2003), pp. 1-16, May 2014.

[14] S.-K. Sul, Control of Electric Machine Drive Systems. Wiley IEEE press, 2011. 